\title{
SATURATION OF THE CLOSED UNBOUNDED FILTER ON THE SET OF REGULAR CARDINALS
}

\author{
BY
}

THOMAS J. JECH AND W. HUGH WOODIN ${ }^{1}$

ABSTRACT. For any $\alpha<\kappa^{+}$, the following are equiconsistent:

(a) $\kappa$ is measurable of order $\alpha$,

(b) $\kappa$ is $\alpha$-Mahlo and the filter $\mathbf{C}[\mathrm{Reg}]$ is saturated.

Introduction. Let $\kappa$ be a regular uncountable cardinal. $\mathbf{C}$ is the filter over $\kappa$ generated by closed unbounded subsets of $\kappa$. If $S \subseteq \kappa$ is a stationary set then

$$
\mathbf{C}[S]=\{C \cap S: C \in \mathbf{C}\}
$$

is the restriction of $\mathbf{C}$ to $S$.

By a result of Solovay $[\mathbf{9}]$, the filter $\mathbf{C}[S]$ is not $\kappa$-saturated for any stationary set $S$; i.e. every stationary set is the union of $\kappa$ disjoint stationary subsets. A natural question is whether the filter $\mathbf{C}$ can be $\kappa^{+}$-saturated, or more generally, whether $\mathbf{C}[S]$ can be $\kappa^{+}$-saturated for some stationary set $S$.

The filter $\mathbf{C}[S]$ is $\kappa^{+}$-saturated if there exists no collection of $\kappa^{+}$stationary subsets of $S$ such that any two of them have nonstationary intersection. To simplify the terminology, we call $\mathbf{C}[S]$ saturated instead of $\kappa^{+}$-saturated.

If $\mathbf{C}$ is saturated then $\kappa$ is a measurable cardinal in an inner model [3]; moreover, if $\kappa$ is a successor cardinal then considerably stronger properties of $\kappa$ can be deduced $[4,7]$. In fact, if $\kappa$ is a successor cardinal and $\kappa>\aleph_{1}$, then $\mathbf{C}$ itself cannot be saturated $[\mathbf{8}]$.

If $\kappa$ is an inaccessible cardinal, let Sing and Reg denote, respectively, the set of all singular and regular cardinals $\alpha<\kappa$. It is not known whether $\mathbf{C}$ [Sing] can be saturated, it is only known that it is then necessary for $\kappa$ to be a measurable cardinal of order $\kappa$ in an inner model. The present paper concerns itself with the saturation of the filter $\mathbf{C}[\mathrm{Reg}]$.

As we assume that the set Reg is stationary, $\kappa$ is a Mahlo cardinal. We show that there is a deep relationship between the hierarchy of Mahlo cardinals on one hand, and the hierarchy [6] of measurable cardinals on the other.

Let $S$ and $T$ be stationary subsets of $\kappa$. Following [2], we let

$$
S<T \quad \text { iff } \text { for almost all } \alpha \in T, S \cap \alpha \text { is a stationary subset of } \alpha \text {. }
$$

"For almost all $\alpha$ " means modulo the filter $\mathbf{C}$. The relation $<$ is a well founded partial ordering. The order of a stationary set is its rank in $<$; the cardinal $\kappa$ is $\alpha$-Mahlo if the length of $<$ restricted to subsets of Reg is at least $\alpha$. Hence 0-Mahlo means inaccessible, 1-Mahlo means Mahlo, 2-Mahlo means that the set of all Mahlo cardinals $<\kappa$ is stationary etc.

Received by the editors October 31, 1984.

1980 Mathematics Subject Classification. Primary 03E55; Secondary 03E35.

${ }^{1}$ Both authors acknowledge support from the National Science Foundation. 
Let $\kappa$ be a measurable cardinal, and let $U$ and $V$ be normal measures on $\kappa$. Following [6], we let

\section{$U \triangleleft V$ iff $U$ belongs to the ultrapower by $V$.}

The relation $\triangleleft$ is a well-founded partial ordering. The order of $U$ is its rank in $\triangleleft ; \kappa$ is $\alpha$-measurable if the length of $\triangleleft$ is at least $\alpha$. Hence 1-measurable means measurable, 2-measurable means that $\kappa$ has a normal measure such that the set of all measurable cardinals $<\kappa$ has measure one, etc.

If $\kappa$ is $\kappa^{+}$-Mahlo it is called greatly Mahlo in $[\mathbf{1}]$. In that case, there is a natural decomposition of $\kappa$ into $\kappa^{+}$almost disjoint stationary sets. Consequently, the filter $\mathbf{C}[\mathrm{Reg}]$ is not saturated.

In this paper we solve the problem of saturation of the filter $\mathbf{C}[\mathrm{Reg}]$ in the case when $\kappa$ is $\alpha$-Mahlo and $\alpha<\kappa^{+}$. We prove equiconsistency of " $\kappa$ is $\alpha$-Mahlo and $\mathrm{C}[\mathrm{Reg}]$ is saturated" with " $\kappa$ is $\alpha$-measurable". More precisely,

THEOREM A. Let $\kappa$ be an $\alpha$-Mahlo cardinal and $0<\alpha<\kappa^{+}$. If the filter $\mathbf{C}[\operatorname{Reg}]$ over $\kappa$ is saturated then $\kappa$ is $\alpha$-measurable in an inner model.

THEOREM B. Let $\kappa$ be a measurable cardinal and $0<\alpha<\kappa^{+}$. If $\kappa$ is $\alpha$ measurable then there is a Boolean-valued model $V^{B}$ in which $\kappa$ is an $\alpha$-Mahlo cardinal and the filter $\mathbf{C}[\mathrm{Reg}]$ over $\kappa$ is saturated.

The proof of Theorem A is implicit in [2] and runs as follows: We assume that $\kappa$ is not $\kappa^{+}$-measurable in any inner model and let $\mathbf{K}$ be the core model for measures [7]. We claim that $\kappa$ is $\alpha$-measurable in $\mathbf{K}$. As in Lemma 4.7 of [2], there is a decomposition $W$ of the set Reg into a maximal almost disjoint collection of $\leq \kappa$ stationary subsets $S$ such that, for each of them, $U_{S}=\mathbf{C}[S] \cap \mathbf{K}$ is a normal measure in the model $\mathbf{K}$. Moreover, the partition $W$ can be obtained such that the length of $<$ restricted to $W$ has length at least $\alpha$. It follows from [2] that if $S$, $T \in W$ and $S<T$, then $\mathbf{K} \models U_{S} \triangleleft U_{T}$. Hence $\kappa$ is $\alpha$-measurable in $\mathbf{K}$.

We devote the rest of our paper to the proof of Theorem B.

Sketch of the forcing construction. We start with a model of ZFC + GCH (the GCH can be made true by a preliminary forcing extension that preserves measurable cardinals and their orders). The idea is to change $\alpha$-measurable cardinals into $\alpha$-Mahlo cardinals, and at the same time, change a measure of order $\beta$ into a saturated filter that coincides with $\mathbf{C}\left[E_{\beta}\right]$ where $E_{\beta}$ is the set of all $\beta$-Mahlo cardinals. And all that while preserving all cofinalities, as well as GCH. In particular, inaccessible cardinals remain inaccessible; nonmeasurable cardinals become non-Mahlo, measurable cardinals of order 1 become Mahlo cardinals, etc.

If $\kappa$ is nonmeasurable in the ground model then the set Reg will become nonstationary; in other words, the set Sing will acquire a closed unbounded subset. If $\kappa$ is measurable of order 1 (in the ground model), we choose a normal measure $U$ on $\kappa$. We arrange things so that each set of measure one will remain stationary in the extension. The set Reg is the union of two sets, $E_{0}=$ the set of all nonmeasurable cardinals and $E_{1}=$ the set of all measurable cardinals, and $E_{0} \in U$. We make the set $E_{1}$ nonstationary. In the process, for every set $A$ of measure one we make sure that $A \cup$ Sing acquires a closed unbounded subset. Hence $\mathbf{C}[\operatorname{Reg}]$ will extend $U$. And we also make sure that $\mathbf{C}[\operatorname{Reg}]$ will be saturated. 
If $\kappa$ is measurable of order 2 then it has a measure $U_{1}$ that concentrates on measurable cardinals of order 1 . We have Reg $=E_{0} \cup E_{1} \cup E_{2}$ where $E_{0}$ is the set of all nonmeasurables, $E_{1}$ is the set of all measurables of order 1 , and $E_{2}=$ all measurables of higher order. Now the idea is to keep $E_{0}$ and $E_{1}$ stationary (while making $E_{2}$ nonstationary) and make both $\mathbf{C}\left[E_{0}\right]$ and $\mathbf{C}\left[E_{1}\right]$ saturated; moreover, all elements of $E_{1}$ will be Mahlo cardinals (formerly being measurable) and so $\kappa$ will be a 2-Mahlo cardinal.

The filter $\mathbf{C}\left[E_{1}\right]$ will extend the measure $U_{1}$ of the ground model. Thus for every $A \in U_{1}, A \cup$ (Sing $\cup E_{0}$ ) acquires a closed unbounded subset. In order to deal with $E_{0}$, we use a measure $U_{0}$ that concentrates on $E_{0}$, and eventually extend $U_{0}$ to $\mathbf{C}\left[E_{0}\right]$. But we do not choose $U_{0}$ arbitrarily; for various reasons $U_{0}$ has to cohere with other steps of our constructions and so $U_{0}$ is the ultrapower by $U_{1}$ of the measures previously chosen on measurable cardinals below $\kappa$.

The basic technique used in our construction involves shooting a closed unbounded set through a given stationary set $A$; our construction is an iteration of this basic technique. The technique is well known and understood: forcing conditions are closed initial segments of the intended closed unbounded set. When the stationary set $S$ contains the set Sing then this notion of forcing does not add bounded subsets of $\kappa$ (see Lemma 1) and that makes the iteration easier to handle.

Our construction is an iteration with Easton support ("backward Easton"). At stage $\kappa$ (where $\kappa$ is an inaccessible cardinal) we perform an iteration $Q$ of length $\kappa^{+}$, with support of size $<\kappa$; we shoot closedc unbounded sets through various stationary subsets of $\kappa$.

If $\kappa$ is nonmeasurable, the iteration $Q$ amounts to nothing more than shooting a club through the set Sing, and then adding $\kappa^{+}$Cohen subsets of $\kappa$. That makes $\kappa$ a non-Mahlo inaccessible cardinal.

If $\kappa$ is measurable of order 1 , we choose a measure $U_{0}$ and iterate shooting a club through sets of the form $A \cup \operatorname{Sing}$ where $A \subseteq E_{0}$ is stationary. The intention is to make the filter $\mathbf{C}\left[E_{0}\right]$ saturated. To achieve that we employ a variation of the Kunen-Paris method $[\mathbf{5}, \mathbf{4}]$. Let $j$ be the elementary embedding given by $U_{0}$. As we perform the iteration, we manage to keep extending $j$ so that the extended embedding is an elementary embedding of the generic extension $V[G]$. This extended embedding $j$ does not live in the generic extension itself, but in a larger extension. However, that larger extension is a $\kappa^{+}$-c.c. extension of $V[G]$ and so $j$ naturally defines a saturated filter over $\kappa$. The construction is so arranged that this filter coincides with $\mathbf{C}\left[E_{0}\right]$.

When $\kappa$ is measurable of higher order, the construction is a more or less natural generalization of the construction outlined above, except that care has to be taken for the various iterations to cohere.

The forcing construction-preliminaries. The basic technique we use is the method of shooting a $c l u b$ (a closed unbounded subset) through a given stationary set. Thus let $\kappa$ be an inaccessible cardinal, and let $S \subseteq \kappa$ be a stationary set. We define a notion of forcing $\mathrm{CU}(S)$ as follows:

$$
\mathrm{CU}(S)=\{p: p \subseteq S,|p|<\kappa \text { and } p \text { is a closed set of ordinals }\} .
$$


A condition $p$ is stronger than $q, p<q$, if $q$ is an initial segment of $p$. A generic filter on $\mathrm{CU}(S)$ yields a closed unbounded set $C$ that is included in $S$ (thus making the complement of $S$ nonstationary).

In general, there is no reason why cardinals or cofinalities should be preserved by this forcing. If, however, $S \supseteq$ Sing, then the forcing is well behaved:

LEMMA 1. If $S \supseteq$ Sing then for every regular $\lambda<\kappa$ there is a dense subset $P \subseteq \mathrm{CU}(S)$ that is $\lambda$-closed.

ProOF. $P=\{p: \sup (p)>\lambda\}$.

It follows that $\mathrm{CU}(S)$ does not add new bounded subsets of $\kappa$ (nor new ordinal sequences of length less than $\kappa$ ) and $\kappa$ remains an inaccessible cardinal. The size of $\mathrm{CU}(S)$ is $\kappa$, and so cardinals above $\kappa$ are also preserved, as is the GCH.

We shall repeatedly apply the forcing notion $\mathrm{CU}(S)$ as follows: Let $E$ be a subset of the set Reg; for instance, $E=E_{0}=$ the set of all regular nonmeasurable cardinals, or $E=E_{\alpha}=$ the set of all measurable cardinals of order $\alpha$. Let $X$ be a subset of $E$. We let

$$
\begin{aligned}
\mathrm{CU}(E, X) & =\{p \subseteq \kappa:|p|<\kappa, p \text { is closed and } p \cap E \subseteq X\} \\
& =\mathrm{CU}(X \cup(\kappa-E)) .
\end{aligned}
$$

As $X \cup(\kappa-E) \supseteq$ Sing, Lemma 1 applies. The club $C$ obtained by this forcing is such that $C \cap E \subseteq X$, i.e. it avoids the set $E-X$. If $X$ is nonstationary then $\mathrm{CU}(E, X)$ makes the set $E$ nonstationary.

Now consider iterations of $\mathrm{CU}(E, X)$. That is, consider an iteration $Q$ of length $\vartheta$ with support of size $<\kappa$, where at stage $\xi$ we force with $\mathrm{CU}(E, X)$ where $X \in V^{Q \mid \xi}$ (while $E \in V$ ). We shall describe a uniform representation of such iterations.

Let $C_{\vartheta}$ be the set of all $p$ of the following form:

(i) $p \subset \vartheta \times \kappa$,

(ii) $|p|<\kappa$,

(iii) $p_{i}=\{\nu<\kappa:(i, \nu) \in p\}$, $\operatorname{supp}(p)=\left\{i<\vartheta: p_{i} \neq \varnothing\right\}$,

$p_{i}$ is a closed set of ordinals $(i<\vartheta)$,

(iv) $p<q$ iff $p \supseteq q$ and each $q_{i}$ is an initial segment of $p_{i}$.

LEMMA 2. If $Q$ is an iteration of length $\vartheta$ as above, then

(a) for every $\lambda<\kappa, Q$ has a dense subset that is $\lambda$-closed,

(b) $Q$ has a dense subset $Q^{\prime}$ isomorphic to a subset of $C_{\vartheta}$, and if $p, q \in Q^{\prime}$ then $p \cup q \in Q^{\prime}$.

PROOF. By simultaneous induction on the length of iteration.

Thus we identify $Q$ with a subset of $C_{\vartheta}$.

It is clear from the definition that the partial ordering $C_{\vartheta}$ satisfies the $\kappa^{+}$-chain condition. As $Q$ is a sublattice of $C_{\vartheta}, Q$ does too, and we state this as

LEMMA 3. $Q$ satisfies the $\kappa^{+}$-chain condition.

Let $X \in V^{Q}$ be a name for a subset of $E$. For each $\nu \in E$, let $W_{\nu}$ be a maximal set of mutually incompatible conditions $p \in Q$ such that $p \Vdash \nu \in X$. As $Q$ has the $\kappa^{+}$-c.c., $W_{\nu}$ has size at most $\kappa$. Let $W \subseteq E \times C_{\vartheta}$ be as follows:

$$
W=\left\{(\nu, p): \nu \in E, p \in W_{\nu}\right\} .
$$


We have $|W| \leq \kappa$; let

$$
\operatorname{supp} W=\bigcup\left\{\operatorname{supp}(p): p \in W_{\nu}, \nu \in E\right\} .
$$

All our iterations will be of length $\leq \kappa^{+}$. In view of the preceding remark, we have a canonical way of describing subsets of $\kappa$ at all stages of all iterations:

DEFinition. A canonical name for a subset of $E \subseteq$ Reg is a set $W \subseteq E \times C_{\kappa^{+}}$ of size $\leq \kappa$.

Given an iteration $Q$ of length $\leq \kappa^{+}$and a generic filter $G$ on $Q$, the $G$ interpretation of a canonical name $W$ is

$$
\{\nu \in E:(\nu, p) \in W \text { for some } p \in G\} \text {. }
$$

In other words, $W$ represents the name $X \in V^{Q}$ such that

$$
\|\nu \in X\|_{Q}=\sum\{p \in Q:(\nu, p) \in W\} \quad(\nu \in E) .
$$

(No matter that not all $p$ involved are conditions in $Q$.)

Now let $\left\{W_{\xi}: \xi<\kappa^{+}\right\}$be a sequence of canonical names. We associate with this sequence the following iteration $Q$ of length $\kappa^{+}$: At stage $\xi$ of the iteration, let $X \in V^{Q 1 \xi}$ be the name represented by the canonical name $W_{\xi}$ (and $E$ the corresponding subset of Reg in the ground model). The $\xi$ th stage of the iteration is the notion of forcing $\mathrm{CU}(E, X) \in V^{Q \uparrow \xi}$. (Of course, we identify each $Q \uparrow \xi$ with a subset of $C_{\kappa^{+}}$.)

Still considering the iteration associated with $\left\{W_{\xi}: \xi<\kappa^{+}\right\}$, let $A$ be a subset of $\kappa^{+}$such that

$$
\operatorname{supp} W_{\xi} \subseteq A \quad \text { whenever } \xi \in A
$$

Let

$$
Q_{A}=\{p \in Q: \operatorname{supp}(q) \subseteq A\} .
$$

Using (*) we can see that $Q_{A}$ is an iteration (indexed by $A$ ) associated with $\left\{W_{\xi}: \xi \in\right.$ $A\}$, and r.o. $\left(Q_{A}\right)$ (the complete Boolean algebra corresponding to $Q_{A}$ ) is a regular subalgebra of r.o. $(Q)$.

Also if $B \subseteq A$ also satisfies (*), then $Q_{B}$ is a regular subalgebra of $Q_{A}$.

The iterations. We shall now describe the forcing construction in detail in the case $\alpha=2$. The general case can be obtained by a suitable modification.

Thus we assume that the ground model $V$ satisfies GCH, and that $\kappa$ is a measurable cardinal of order 2 . Let

$$
\begin{aligned}
& E_{0}=\text { the set of all inaccessible nonmeasurable cardinals below } \kappa, \\
& E_{1}=\text { the set of all measurable cardinals below } \kappa .
\end{aligned}
$$

Let $U_{1}$ be a normal measure on $\kappa$ concentrating on $E_{1}$. For each $\delta \in E_{1}$ let $U_{\delta}$ be a normal measure on $\delta$ concentrating on $E_{0} \cap \delta$, and let $U_{0}$ be the measure on $\kappa$ represented in the $U_{1}$-ultrapower by $\left\langle U_{\alpha}: \alpha<\kappa\right\rangle$.

The notion of forcing the $P$ we are to construct is an iteration of length $\kappa+1$, with Easton support. That is, we take direct limits at regular stages, and inverse limits otherwise.

All cardinals and cofinalities are preserved at each step of this construction, as is the GCH. 
A nontrivial iteration occurs only at inaccessible stages; when $\delta$ is not inaccessible than $P_{\delta+1}=P_{\delta}$. The final notion of forcing is $P=P_{\kappa+1}$.

When $\delta$ is inaccessible, then $P_{\delta+1}=P_{\delta} * Q_{\delta}$ where $Q_{\delta}$ is, in $V^{P_{\delta}}$, a notion of forcing of the form $Q_{A}$, where $Q$ is the iteration (of length $\delta^{+}$with $<\delta$ support) associated with some $\delta^{+}$-sequence of canonical terms, and $A$ is a suitable subset of $\delta^{+}$that satisfies $(*)$.

We define $Q_{\delta} \in V^{P}$ by induction on $\delta$, depending on whether $\delta \in E_{0}, \delta \in E_{1}$ or $\delta=\kappa$. In each case we use a certain $\delta^{+}$-sequence of canonical names $\left\langle W_{\xi}: \xi<\delta^{+}\right\rangle$. Each sequence has these properties:

(i) if $\xi$ is even then $W_{\xi}$ is a name for a subset of $E_{0} \cap \delta$; if $\xi$ is odd then $W_{\xi}$ is a name for a subset of $E_{1} \cap \delta$,

(ii) the sequence enumerates all canonical names of subsets of $E_{0} \cap \delta$ and $E_{1} \cap \delta$; moreover,

(iii) each canonical name appears $\delta^{+}$times.

Case I: $\delta \in E_{0}$. In this case ( $\delta$ not measurable) we choose any $\delta^{+}$-sequence of canonical names with properties (i), (ii), (iii) and let $Q_{\delta}=Q$ be the iteration associated with the sequence.

Note that because both $\mathrm{CU}\left(E_{0}, \varnothing\right)$ and $\mathrm{CU}\left(E_{1}, \varnothing\right)$ appear in the iteration, both $E_{0}$ and $E_{1}$ become nonstationary and hence the set of all singular cardinals below $\delta$ has a closed unbounded subset in $V^{P_{\delta+1}}$. The result is that $\delta$, while it remains inaccessible, is a non-Mahlo cardinal in $V^{P_{\delta+1}}$ (and therefore in $V^{P}$ ).

We note in passing that the notion of forcing $Q$ is the same as a two step iteration, first shooting a closed unbounded set through Sing and then adding $\delta^{+}$ Cohen subsets of $\delta$.

Case II: $\delta \in E_{1}$. We have chosen a normal measure $U_{\delta}$ on $\delta$ that concentrates on $E_{0} \cap \delta$. Let $j$ denote the elementary embedding $j: V \rightarrow M$ where $M=$ the ultrapower of $V$ by $U_{\delta}$. In the model $M, j\left(P_{\delta}\right)$ is an iteration of length $j(\delta)$, with Easton support, and can be factored out as

$$
j\left(P_{\delta}\right)=P_{\delta} * \tilde{Q}_{\delta} * R
$$

where $\tilde{Q}_{\delta} \in M^{P}$ is the forcing done at stage $\delta$, and $R$ has a dense subset that is $\delta$-closed. In $M, \delta$ is a nonmeasurable cardinal (because $E_{0} \in U_{\delta}$ ) and so $\tilde{Q}_{\delta}$ is, in $M^{P_{\delta}}$, the iteration of length $\delta^{+}$associated with some $\delta^{+}$-sequence $\left\langle W_{\xi}: \xi<\delta^{+}\right\rangle$of canonical names.

Because $M$ is an ultrapower of $V$ and because $\left|P_{\delta}\right|=\delta$, it follows that $M^{P_{\delta}}$ has the same ordinal $\delta$-sequences as $V^{P_{\delta}}$. Hence $\left\langle W_{\xi}: \xi<\delta^{+}\right\rangle$is a sequence of canonical names in $V^{P_{\delta}}$ satisfying (i), (ii), (iii), and $Q=\tilde{Q}_{\delta}$ is the associated iteration of length $\delta^{+}$(in $\left.V^{P_{\delta}}\right)$. Thus we have defined $Q$.

We shall now define $A \subseteq \delta^{+}$. We work inside the model $V_{1}=V^{P_{\delta}}$ (or, equivalently, we choose some generic filter $G_{1} \subset P$ and let $\left.V_{1}=V\left[G_{1}\right]\right)$.

LEMMA 4. The elementary embedding $j: V \rightarrow M$ can be extended to an elementary embedding $j: V_{1} \rightarrow M_{1}$ that lives in $V_{1}^{Q}$.

PROOF. It is enough to find in $V_{1}^{Q}$ a filter $H_{1} \subseteq R$ generic over $M^{P_{\delta} * Q}$. Then, if $G_{1} \subset P$ is $V$-generic and $G \subset Q$ is $V\left[G_{1}\right]$-generic, we let $j\left(G_{1}\right)=G_{1} \times G \times H_{1}$ and extend $j$ to $V_{1}=V\left[G_{1}\right]$ accordingly. The forcing notion $R$ has a dense subset that is $\delta$-closed. Moreover, the size of the power set of $R$ (in $M^{P_{\delta}^{*} Q}$ ) is $j\left(\delta^{+}\right)$, and 


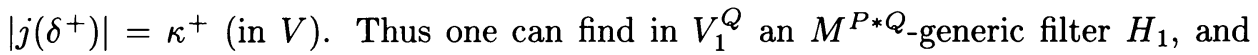
consequently extend $j$ to an elementary embedding $j: V_{1} \rightarrow M_{1}$ where

$$
M_{1}=M^{P_{\delta} * Q}\left[H_{1}\right] .
$$

We construct $A \subseteq \delta^{+}$by induction, that is we construct $A \cap \alpha$ for $\alpha<\delta^{+}$. Simultaneously, we choose on the side $V_{1}^{Q}$-names $q_{\alpha}$ for conditions in $j\left(Q_{A \cap \alpha}\right)$. The purpose of these conditions is to produce, in $V_{1}^{Q}$, an $M_{1}$-generic filter $H$ on $j\left(Q_{A}\right)$, thus making it possible to further extend $j$ to an elementary embedding $j: V_{1}^{Q_{A}} \rightarrow M_{1}[H]$.

The conditions $q_{\alpha}, \alpha<\delta^{+}$, will form a descending chain. In order for the induction to continue at limit stages, we require that, for each $\xi \in \operatorname{supp}\left(q_{\alpha}\right)$, the supremum of the $\xi$ th coordinate of $q$ is at least $\delta$.

In $V_{1}^{Q}$, let $G$ be the canonical generic filter on $Q ; G$ yields a sequence $\left\langle C_{\xi}: \xi<\delta\right\rangle$ of closed unbounded subsets of $\delta$. If $q$ is a condition in $j(Q)$ and $\eta<j(\delta)$ then the $\eta$ th coordinate of $q$ is a closed bounded subset of $j(\delta)$. We make a further requirement for each $\alpha<\delta^{+}$:

$(* *) \quad$ For each $\xi \in A \cap \alpha$, the $j(\xi)$ th coordinate of $q_{\alpha}$ extends $C_{\xi}$.

Note that $(* *)$ entails that $\delta$ is a member of the $j(\xi)$ th coordinate of $q_{\alpha}$.

In order to have the $q_{\alpha}$ produce an $M_{1}$-generic filter, we choose an enumeration $\left\langle a_{\alpha}: \alpha<\delta^{+}\right\rangle$of all $V_{1}^{Q}$-names for an antichain of $j(Q)$ in $M_{1}$, such that each $a_{\alpha}$ occurs $\delta^{+}$many times. This is possible because of the size of $j(Q)$ and because $V_{1}^{Q}$ is a $\kappa^{+}$-c.c. extension of $V_{1}$.

Suppose that $A \cap \xi$ and $q_{\xi}$ have been constructed for all $\xi<\alpha$. If $\alpha$ is a limit than $A \cap \alpha=\bigcup_{\xi<\alpha}(A \cap \xi)$ and we find a $Q$-name for a condition $q_{\alpha}$ in $j\left(Q_{A \cap \alpha}\right)$ below the $q_{\xi}$ 's such that $q_{\alpha}$ is below $a_{\alpha}$ if possible ( $a_{\alpha}$ may not be maximal, or its elements may not all be in $j\left(Q_{A \cap \alpha}\right)$ ). We also make sure that $q_{\alpha}$ satisfies the inductive requirement on the supremums (the requirement $(* *)$ is satisfied automatically).

Now suppose that $A \cap \alpha$ and $q_{\alpha}$ have been defined; we are to find $q_{\alpha}$ and to decide whether $\alpha \in A$. Here we distinguish between the case when $\alpha$ is even and the case when $\alpha$ is odd. However, in either case we do not let $\alpha \in A$ unless $\operatorname{supp}\left(W_{\alpha}\right) \subseteq A \cap \alpha$, where $W_{\alpha}$ is the $\alpha$ th canonical name in the definition of $Q$. This is to insure that $A$ satisfies (*). If $\operatorname{supp}\left(W_{\alpha}\right) \nsubseteq \subset A \cap \alpha$ then we let $A \cap(\alpha+1)=A \cap \alpha$, and choose a $Q$-name for $q_{\alpha+1} \in j\left(Q_{A \cap \alpha}\right)$ below $q_{\alpha}$ such that $q_{\alpha+1}$ is below $a_{\alpha+1}$ if possible (and satisfies the inductive requirements).

If $\operatorname{supp}\left(W_{\alpha}\right) \subseteq A \cap \alpha$ then we distinguish between $\alpha$ odd and $\alpha$ even. We recall that $E_{0} \in U_{0}$ and therefore $\delta \in j\left(E_{0}\right), \delta \notin j\left(E_{1}\right)$.

Case A: $\alpha$ odd. We let $\alpha \in A$. This means that we let $Q_{A}$ shoot a club through $W_{\alpha} \cup\left(\kappa-E_{1}\right)$. As we do this for all odd $\alpha$, we particularly include $\operatorname{CU}\left(E_{1}, \varnothing\right)$ in the iteration and therefore make $E_{1} \cap \delta$ nonstationary.

We also choose a $Q$-name $q_{\alpha+1} \in j\left(Q_{A \cap(\alpha+1)}\right)$ below $q_{\alpha}$ such that $q_{\alpha+1}$ is below $a_{\alpha+1}$ if possible and satisfies the inductive requirements. In order to satisfy $(* *)$ it is necessary that $C_{\alpha} \cup\{\delta\}$ is the $j(\alpha)$ th coordinate of a condition in $j(Q)$. In other words, $C_{\alpha} \cup\{\delta\}$ has to be a condition in $\mathrm{CU}\left(j\left(E_{1} \cap \delta\right), j\left(W_{\alpha}\right)\right)$. But every initial segment of $C_{\alpha}$ is a condition in $\mathrm{CU}\left(E_{1}, W_{\alpha}\right)$ because it is the $\alpha$ th coordinate of a condition in $Q$, and so the only question is whether $C_{\alpha} \cup\{\delta\} \subseteq j\left(W_{\alpha} \cup\left(\delta-E_{1}\right)\right)$. But, as we remarked, $\delta \notin j\left(E_{1}\right)$, and so it is possible to satisfy $(* *)$. 
Case B: $\alpha$ even. First we choose a $Q$-name $q$ for a condition in $j\left(Q_{A \cap \alpha}\right)$ below $q_{\alpha}$ such that, in $V_{1}^{Q}, q$ decides $\delta \in j\left(W_{\alpha}\right)$. That is, we have

$$
\llbracket \text { either } q \Vdash \delta \in j\left(W_{\alpha}\right) \text {, or } q \Vdash \delta \notin j\left(W_{\alpha}\right) \rrbracket_{Q}=1 \text {. }
$$

If $\llbracket q \Vdash \delta \in j\left(W_{\alpha}\right) \rrbracket_{Q} \neq 1$, then we let $A \cap(\alpha+1)=A \cap \alpha$, and choose $q_{\alpha+1}$ below $q$, below $a_{\alpha+1}$ if possible, subject to the inductive requirement. If $\llbracket q \Vdash \delta \in$ $j\left(W_{\alpha}\right) \rrbracket_{Q}=1$, then we let $\alpha \in A$, and fine $q_{\alpha+1} \in j\left(Q_{A \cap(\alpha+1)}\right)$ below $q$, below $a_{\alpha+1}$ if possible, and such that $q_{\alpha+1}$ satisfies the inductive requirements.

Arguing in $V_{1}^{Q}$, we use the fact that $q \Vdash \delta \in j\left(W_{\alpha}\right)$. Hence $q \in M_{1}^{j\left(Q_{A \cap \alpha}\right)}$ forces

$$
C_{\alpha} \cup\{\delta\} \in \mathrm{CU}\left(j\left(E_{0} \cap \delta\right), j\left(W_{\alpha}\right)\right)
$$

and hence there is a condition in $j\left(Q_{A \cap(\alpha+1)}\right)$ that extends $q$ and whose $j(\alpha)$ th coordinate extends $C_{\alpha}$. Thus a $q_{\alpha+1}$ can be found that satisfies (**).

This completes the construction of $A \subseteq \delta^{+}$, and we let $Q_{\delta}=Q_{A}$, and $P_{\delta+1}=$ $P_{\delta} * Q$.

Since every maximal antichain of $j\left(Q_{A}\right)$ appears in the sequence $\left\langle a_{\alpha}: \alpha<\delta^{+}\right\rangle$, the conditions $q$ yield (in $V_{1}^{Q}$ ) an $M_{1}$-generic filter $H$ on $j\left(Q_{A}\right)$. Moreover, for each $\alpha \in A$ the club $C_{\alpha} \subseteq \delta$ is an initial segment of the $j(\alpha)$ th coordinate of $H$. Thus if we let $j(G \nmid A)=H$, we get an extension of the elementary embedding $j: V_{1} \rightarrow M_{1}$ to an elementary embedding

$$
j: V_{1}[G \uparrow A] \rightarrow M_{1}[H],
$$

in other words to an elementary embedding $j$ of $V_{1}^{Q_{A}}$ (and $j$ lives in $V_{1}^{Q}$ ).

Let $Q / Q_{A}$ denote the complete Boolean algebra (in $V_{1}^{Q_{A}}$ ) such that $Q_{A} *\left(Q / Q_{A}\right)=Q$. As both $Q$ and $Q_{A}$ satisfy the $\kappa^{+}$-chain condition, $Q / Q_{A}$ does as well. The elementary embedding $j$ of $V_{1}^{Q_{A}}$ in $V^{Q}$ produces naturally a filter $F \in V_{1}^{Q_{A}}$ on $\delta$ :

$x \in F$ iff every condition in $Q / Q_{A}$ forces $\delta \in j(X)$.

As $Q / Q_{A}$ has the $\kappa^{+}$-c.c., $F$ is $\kappa^{+}$-saturated (in $V_{1}^{Q_{A}}$ ). In fact, if we let (in $V_{1}^{Q_{A}}$ )

$$
e(X)=\|\delta \in j(X)\|_{Q / Q_{A}} \quad(X \subseteq \delta),
$$

then $e$ is an embedding of the Boolean algebra $P(\delta) / F$ into $Q / Q_{A}$.

Working in $V_{1}^{Q_{A}}$, we show that $F=\mathbf{C}\left[E_{0} \cap \delta\right]$. The definition of $F$ can be restated as follows:

$$
\begin{aligned}
X \in F \quad & \text { iff } \llbracket \exists q \in H q \Vdash \delta \in j(X) \rrbracket_{Q}=1, \\
& \text { iff } \exists \alpha \llbracket q_{\alpha} \Vdash \delta \in j(X) \rrbracket_{Q}=1 .
\end{aligned}
$$

It is clear that every closed unbounded set belongs to $F$. It is also easy to see that $E_{0} \cap \delta \in F$ (because $\left.\delta \in j\left(E_{0} \cap \delta\right)\right)$. Thus $F \supseteq \mathbf{C}\left[E_{0} \cap \delta\right]$.

If $X \in F$ then there is a sufficiently large $\alpha$ such that $W_{\alpha}$ is a name for $F$, $\operatorname{supp}\left(W_{\alpha}\right) \subseteq A \cap \alpha$, and $\llbracket q_{\alpha} \Vdash \delta \in j(X) \rrbracket_{Q}=1$. Then $\alpha \in A$, and $C_{\alpha}$ is a closed unbounded subset of $X \cup\left(\delta-E_{0}\right)$. Hence there is a club $C_{\alpha} \in V_{1}^{Q_{A}}$ such that $C_{\alpha} \cap E_{0} \subseteq X$, and so $X \in \mathbf{C}\left[E_{0} \cap \delta\right]$. Therefore, $F=\mathbf{C}\left[E_{0} \cap \delta\right]$.

Thus $\mathbf{C}\left[E_{0} \cap \delta\right]$ is saturated and $\delta$ is a Mahlo cardinal, in $V^{P_{\delta+1}}$. As further iteration does not add $\delta$-sequences, $\delta$ will remain a Mahlo cardinal. 
Case III: $\delta=\kappa$. So far, we have constructed $P_{\kappa}$, an iteration of length $\kappa$. Let $V_{1}=V^{P_{\kappa}}$ (or $V_{1}=V\left[G_{1}\right]$ where $G_{1}$ is a generic filter on $\left.P_{\kappa}\right)$. In $V_{1}$, both $E_{0}$ and $E_{1}$ are stationary sets, every element of $E_{0}$ is 0 -Mahlo (inaccessible and non-Mahlo) and every element of $E_{1}$ is 1-Mahlo. We shall construct $Q_{\kappa}$ so that in $V_{1}^{Q_{\kappa}}=V^{P_{\kappa} * Q_{\kappa}}=V^{P}, E_{0}$ and $E_{1}$ remain stationary, and both $\mathbf{C}\left[E_{0}\right]$ and $\mathbf{C}\left[E_{1}\right]$ are saturated. Thus in $V^{P}, \kappa$ is a 2-Mahlo cardinal and $\mathbf{C}[\mathrm{Reg}]$ is saturated.

$U_{0}$ and $U_{1}$ are the two measures on $\kappa$ introduced previously, and $j^{0}: V \rightarrow M^{0}$ and $j^{1}: V \rightarrow M^{1}$ are the corresponding elementary embeddings.

First we define $Q$. It is the iteration of closed unbounded forcing of length $\kappa^{+}$, associated with a $\kappa^{+}$-sequence $\left\langle W_{\alpha}: \alpha<\kappa^{+}\right\rangle$of canonical names. We let $\left\langle W_{\alpha}\right\rangle$ be the sequence in the ultrapower $M^{0}$ determined by the $\delta^{+}$-sequences we have chosen at stages $\delta \in E_{0}$. Because $M^{0} \subseteq M^{1} \subseteq V$ and $\left|P_{\kappa}\right|=\kappa$, the definition of $Q$ from $\left\langle W_{\alpha}\right\rangle$ is the same in each $M^{0}\left[G_{1}\right], M^{1}\left[G_{1}\right]$ and $V\left[G_{1}\right]$.

Working in $V_{1}$, we now construct a set $A_{1} \subseteq \kappa^{+}$so as to take $Q_{\delta}=Q_{A_{1}}$. In order to satisfy the condition

$$
\operatorname{supp}\left(W_{\alpha}\right) \subseteq A_{1} \quad \text { whenever } \alpha \in A_{1},
$$

we put $\alpha \in A_{1}$ only if supp $W_{\alpha} \subseteq A_{1} \cap \alpha$. It follows from the way we construct $A_{1}$ that the saturation of $\mathbf{C}\left[E_{0}\right]$ is guaranteed by the iteration below $\kappa$, and the saturation of $\mathbf{C}\left[E_{1}\right]$ will be achieved by a side construction of a sequence $\left\{q_{\alpha}\right\}$ of names for conditions. We recall that if $\alpha$ is even than $W_{\alpha}$ is a canonical name for a subset of $E_{0}$ (and the $\alpha$ th stage in the iteration $Q$ has the form $\mathrm{CU}\left(E_{0}, W_{\alpha}\right)$ ), and if $\alpha$ is odd then $W_{\alpha}$ is a name for a subset of $E_{1}$.

We use the embedding $j^{1}: V \rightarrow M^{1}$. We have $j^{1}\left(P_{\kappa}\right)=P_{\kappa} * Q_{A_{0}} * R$ where $R$ has a dense $\kappa$-closed subset, and $A_{0} \in M^{1}\left[G_{1}\right]$ is a subset of $\kappa^{+}$. As $\kappa \in j^{1}\left(E_{1}\right)$, the set $A_{0}$ is constructed in $M^{1}$ as described in the case $\delta \in E_{1}$. We construct $A_{1}$ so that $A_{1} \subseteq A_{0}$.

As in Lemma 4, the elementary embedding $j^{1}: V \rightarrow M^{1}$ extends to an elementary embedding $j: V_{1} \rightarrow M_{1}^{1}$ that lives in $V_{1}^{Q}$. The embedding is defined by $j\left(G_{1}\right)=$ $G_{1} \times\left(G \uparrow A_{0}\right) \times H_{1}$, where $H_{1}$ is a $M^{1}\left[G_{1}\right]\left[G \uparrow A_{0}\right]$-generic filter on $R$.

Along with $A_{1} \subseteq A_{0}$ we construct the sequence $\left\langle q_{\alpha}: \alpha<\kappa^{+}\right\rangle$. Each $q_{\alpha}$ is a $V_{1}^{Q}$-name for a condition in $j\left(Q_{A \cap \alpha}\right)$. The conditions form a descending chain; we require that, for each $\xi \in \operatorname{supp}\left(q_{\alpha}\right)$, the supremum of the $\xi$ th coordinate of $q_{\alpha}$ is at least $\kappa$. We further require:

For each $\xi \in A \cap \alpha$, the $j(\xi)$ th coordinate of $q_{\alpha}$ extends $C_{\xi}$.

Here $C_{\xi}$ is the club $\subseteq \kappa$ given by the $\xi$ th coordinate of $G$. We also choose an enumeration $\left\langle a_{\alpha}: \alpha<\kappa^{+}\right\rangle$of all $V_{1}^{Q}$-names for antichains of $j(Q)$ in $M_{1}^{1}$ such that each $a_{\alpha}$ occurs $\kappa^{+}$many times.

Suppose that $A_{1} \cap \xi$ and $q_{\xi}$ have been defined for all $\xi<\alpha$. If $\alpha$ is a limit then $A_{1} \cap \alpha=\bigcup_{\xi<\alpha}\left(A_{1} \cap \xi\right)$ and we find a $Q$-name for $q_{\alpha} \in j\left(Q_{A_{1} \cap \alpha}\right)$ below the $q_{\xi}$ 's such that $q_{\alpha}$ is below $a_{\alpha}$ if possible, and such that $q_{\alpha}$ satisfies the inductive requirement.

Now suppose that $A_{1} \cap \alpha$ and $q_{\alpha}$ have been constructed. If $\operatorname{supp}\left(W_{\alpha}\right) \nsubseteq A_{1} \cap \alpha$ or if $\alpha \notin A_{0}$ then we let $A_{1} \cap(\alpha+1)=A_{1} \cap \alpha$, and choose $q_{\alpha+1} \in j\left(Q_{A_{1} \cap \alpha}\right)$ below $q_{\alpha}$, below $a_{\alpha+1}$ if possible, subject to the inductive requirement.

If supp $\left(W_{\alpha}\right) \subseteq A_{1} \cap \alpha$ and $\alpha \in A_{0}$, then there are two cases: $\alpha$ even and $\alpha$ odd. 
Case A: $\alpha$ even. We let $\alpha \in A_{1}$. We choose a $Q$-name $q_{\alpha+1} \in j\left(Q_{A_{1} \cap(\alpha+1)}\right)$ below $q_{\alpha}$, below $a_{\alpha+1}$ if possible, such that $q_{\alpha+1}$ satisfies the inductive requirements. Since $\kappa \notin j\left(E_{0}\right)$, it is possible to satisfy $(* *)$.

Case B: $\alpha$ odd. We choose a $Q$-name $q$ for a condition in $j\left(Q_{A_{1} \cap \alpha}\right)$ below $q_{\alpha}$ such that in $V_{1}^{Q}, q$ decides $\delta \in j\left(W_{\alpha}\right)$. If $\llbracket q \Vdash \delta \in j\left(W_{\alpha}\right) \rrbracket_{Q} \neq 1$, then we let $A_{1} \cap(\alpha+1)=A_{1} \cap \alpha$, and choose $q_{\alpha+1}$ below $q$, below $a_{\alpha+1}$ if possible, subject to the inductive requirement.

If $\llbracket q \Vdash \delta \in j\left(W_{\alpha}\right) \rrbracket_{Q}=1$, then we let $\alpha \in A_{1}$, and find $q_{\alpha+1} \in j\left(Q_{A_{1} \cap(\alpha+1)}\right)$ below $q$, below $a_{\alpha+1}$ if possible, and such that $q_{\alpha+1}$ satisfies the inductive requirements. The same argument we used in the case $\delta \in E_{1}, \alpha$ even, shows that a $q_{\alpha+1}$ can be found that satisfies $(* *)$.

This completes the construction of $A_{1} \subseteq A_{0}$, and we let $Q_{\kappa}=Q_{A_{1}}$, and $P_{\kappa}=$ $P_{\kappa+1}=P_{\kappa} * Q_{\kappa}$. We have to show that both $\mathbf{C}\left[E_{0}\right]$ and $\mathbf{C}\left[E_{1}\right]$ are saturated in $V^{P}=V_{1}^{Q_{A_{1}}}$.

\section{Saturation of $\mathbf{C}\left[E_{1}\right]$.}

The conditions $q_{\alpha}$ produce (in $V_{1}^{Q}$ ) an $M_{1}^{1}$-generic filter $H$ on $j\left(Q_{A_{1}}\right)$. For each $\alpha \in A_{1}$, the club $C_{\alpha} \subseteq \kappa$ is an initial segment of the $j(\alpha)$ th coordinate of $H$ and so if we let $j\left(G \mid A_{1}\right)=H$, we can extend the elementary embedding $j: V_{1} \rightarrow M_{1}^{1}$ to an elementary embedding of $V_{1}^{Q_{A_{1}}}$. For $X \subseteq \delta$ in $V_{1}^{Q_{A_{1}}}$, let

$$
X \in F \text { iff every condition in } Q / Q_{A_{1}} \text { forces } \delta \in j(X) .
$$

The filter $F$ is $\kappa^{+}$-saturated, and clearly $\mathbf{C}\left[E_{1}\right] \subseteq F$.

The proof that $F=\mathbf{C}\left[E_{1}\right]$ is as in the case of $\mathbf{C}\left[E_{0} \cap \delta\right]$, but with a little twist. Let $X \in F$. There is a sufficiently large odd $\alpha$ such that $W_{\alpha}$ is a name for $F$, $\operatorname{supp}\left(W_{\alpha}\right) \subseteq A_{1} \cap \alpha$, and $\llbracket q_{\alpha} \Vdash \delta \in j(X) \rrbracket_{Q}=1$. Because of the definition of $A_{0}$ in $M_{1}^{P_{\kappa}}$ (see the case $\delta \in E_{1}, \alpha$ odd), we have $\alpha \in A_{0}$. Hence $\alpha \in A_{1}$, and so there is a club $C_{\alpha} \in V_{1}^{Q_{A_{1}}}$ such that $C_{\alpha} \cap E_{1} \subseteq X$, and so $X \in \mathbf{C}\left[E_{1}\right]$. Therefore, $F=\mathbf{C}\left[E_{1}\right]$.

\section{Saturation of $\mathbf{C}\left[E_{0}\right]$.}

We want to show that in $V_{1}^{Q_{A_{1}}}$, the filter $\mathbf{C}\left[E_{0}\right]$ is saturated. We shall argue in several steps: one, in $\left(M^{1}\right)^{P_{\kappa} * Q_{A_{0}}}, \mathbf{C}\left[E_{0}\right]$ is saturated; two, in $V_{1}^{Q_{A_{0}}}, \mathbf{C}\left[E_{0}\right]$ is saturated; three, in $V^{Q_{A_{1}}}, \mathbf{C}\left[E_{0}\right]$ is saturated. Of course, $\mathbf{C}\left[E_{0}\right]$ is a different filter in each model.

The iteration $Q_{A_{0}}$ in $\left(M^{1}\right)^{P_{\kappa}}$ has been constructed so that $\mathbf{C}\left[E_{0}\right]$ is saturated in $\left(M^{1}\right)^{P_{\kappa} * Q_{A_{0}}}$; the construction is the $U_{1}$-ultrapower of the construction we performed on all $\delta \in E_{1}$. Because $M_{1}$ is an ultrapower of $V$, and $P_{\kappa} * Q_{A_{0}}$ has the $\kappa^{+}$-c.c., the model $\left(M^{1}\right)^{P_{\kappa} * Q_{A_{0}}}$ is closed under $\kappa$-sequences in $V_{1}^{Q_{A_{0}}}$. Thus $\mathbf{C}\left[E_{0}\right]$

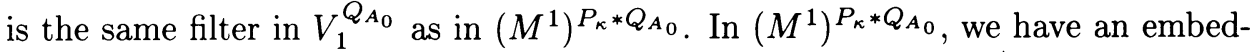
ding $e$ of the algebra $P(\kappa) / \mathbf{C}\left[E_{0}\right]$ into $Q / Q_{A_{0}}$. But $Q / Q_{A_{0}}$ is $\kappa^{+}$-saturated even in $V_{1}^{Q_{A_{0}}}$. Hence $\mathbf{C}\left[E_{0}\right]$ is a saturated filter in $V_{1}^{Q_{A_{0}}}$.

As $A_{1} \subseteq A_{0}$, we have $V_{1}^{Q_{A_{1}}} \subseteq V_{1}^{Q_{A_{0}}}$ and there may be stationary sets in $V_{1}^{Q_{A_{1}}}$ that are nonstationary in $V_{1}^{Q_{A_{0}}}$. However, we prove the following:

LEMMA 5. We work in $V_{1}^{Q_{A_{1}}}$. For every $X \subseteq E_{0}$, if $X$ is stationary, then $\llbracket X$ is stationary $\rrbracket_{Q_{A_{0}} / Q_{A_{1}}} \neq 0$. 
We give a proof of the lemma below. Granted Lemma 5, we argue as follows, in $V^{Q_{A_{1}}}$ : If the filter $\mathbf{C}\left[E_{0}\right]$ is not saturated, there is an almost disjoint family $\left\{S_{\xi}: \xi<\kappa^{+}\right\}$of stationary sets. Because in $V^{Q_{A_{0}}}, \mathbf{C}\left[E_{0}\right]$ is saturated, we have

$$
\llbracket \exists \eta \forall \xi \geq \eta S_{\xi} \text { is nonstationary } \rrbracket_{Q_{A_{0}} / Q_{A_{1}}}=1 .
$$

But $Q_{A_{0}} / Q_{A_{1}}$ has the $\kappa^{+}$-c.c. and therefore

$$
\exists \eta \forall \xi \geq \eta \llbracket S_{\xi} \text { is nonstationary } \rrbracket=1 .
$$

And that contradicts Lemma 5.

PROOF OF LEMMA 5. We want to prove that if $X \subseteq E_{0}$ and if $\llbracket X$ is nonstationary $\rrbracket=1$ then $X$ is nonstationary. Equivalently, work in $V_{1}$ and let $X \in V_{1}^{Q_{A_{1}}}$ be such that $\llbracket X \subseteq E_{0} \rrbracket_{Q_{A_{1}}}=1$. Let $\sigma$ be the sentence

"there is a club $C$ such that $C \cap E_{0} \subseteq X$ ".

It is enough to show that

$$
\llbracket \sigma \rrbracket_{Q_{A_{0}}}=1 \text { implies } \llbracket \sigma \rrbracket_{Q_{A_{1}}}=1 .
$$

Thus let $X \in V_{1}^{Q_{A_{1}}}$ be a name for a subset of $E_{0}$. There are arbitrarily large even $\alpha<\kappa^{+}$such that the canonical name $W_{\alpha}$ is a name for $X$, and supp $W_{\alpha} \subseteq A_{1}$. Because $\|\sigma\|_{Q_{A_{0}}}=1$, there is a canonical name $C$ for a club such that $C \in V_{1}^{Q_{A_{0}}}$, $\operatorname{supp}(C) \subseteq A_{0}$, and $\llbracket C \cap E_{0} \subseteq X \rrbracket_{Q_{A_{0}}}=1$. Let $\beta$ be an upper bound for $\operatorname{supp}(C)$.

Let $\alpha \geq \beta$ be such that $W_{\alpha}$ is a name for $X$ and $\operatorname{supp}\left(W_{\alpha}\right) \subseteq A_{1}$. We look at the construction of $A_{0}$ in $\left(M^{1}\right)^{P_{\kappa}}$. First, we have $C \in\left(M^{1}\right)^{P_{\kappa} * Q_{A_{0}}}$; since $\operatorname{supp}(C) \subseteq \beta \leq \alpha$, we have in fact $C \in\left(M^{1}\right)^{P_{\kappa} * Q_{A_{0} \cap \alpha}}$, and

$$
\left(M^{1}\right)^{P_{\kappa}} \Vdash \llbracket C \cap E_{0} \subseteq W_{\alpha} \rrbracket_{Q_{A_{0} \cap \alpha}}=1 .
$$

According to the definition of $A_{0}$ in $\left(M^{1}\right)^{P_{\kappa}}$ (see the case $\delta \in E_{1}, \alpha$ even), this guarantees that $\alpha \in A_{0}$. Hence by the definition of $A_{1}$ ( $\alpha$ even), we have $\alpha \in A_{1}$, and consequently $\llbracket \sigma \rrbracket_{Q_{A_{1}}}=1$.

The general case. The construction of $P$ for a 2-measurable cardinal generalizes to measurable cardinals of higher order. We shall very briefly outline the construction of $Q_{\kappa}$ for the case of a measurable cardinal of order $\omega$.

We have normal measures $U_{0} \triangleleft U_{1} \triangleleft U_{2} \triangleleft \cdots \triangleleft U_{n} \triangleleft \cdots$ on $\kappa$, concentrating on sets $E_{0}, E_{1}, E_{2}, \ldots, E_{n}, \ldots$ where $E_{n}$ is the set of all $n$-measurable cardinals. Instead of even and odd, we divide ordinals $<\kappa^{+}$into ordinals of type $n$, for $n \in \omega$. We have elementary embeddings $j^{n}: V \rightarrow M^{n}$ corresponding to the ultrapowers by the $U_{n}$. We let $\left\langle W_{\alpha}: \alpha<\kappa^{+}\right\rangle$be the sequence of canonical names (of subsets of the $\left.E_{n}\right)$ given by the embedding $j^{0}$. Thus $j^{0}\left(P_{\kappa}\right)=P_{\kappa} * Q * R_{0}$, where $Q$ is the iteration associated with $\left\langle W_{\alpha}\right\rangle$. For each $n$ we have

$$
j^{n+1}\left(P_{\kappa}\right)=P_{\kappa} * Q_{A_{n}} * R_{n+1}
$$

where $A_{n} \in\left(M^{n+1}\right)^{P_{\kappa}}$ and $\kappa^{+} \supseteq A_{0} \supseteq A_{1} \supseteq \cdots \supseteq A_{n} \supseteq \cdots$.

We construct $A=A_{\omega}$ as follows: If $\alpha$ is an ordinal of type $n$ then we let $a \in A_{\omega}$ just in case $\operatorname{supp}\left(W_{\alpha}\right) \subseteq A_{n} \cap \alpha$, and $\alpha \in A_{n}$. Because of the construction of $P_{\kappa}$, if $\alpha$ satisfies these two conditions, we also have $\alpha \in A_{n+1}, \alpha \in A_{n+2}$, etc. Hence $A_{\omega} \subseteq \bigcap_{n=0}^{\infty} A_{n}$. 
In this case when the order of $\kappa$ is a limit ordinal, we do not have to construct the side conditions. To show that $\mathbf{C}\left[E_{n}\right]$ is saturated in $V_{1}^{Q_{A_{1}}}$, we argue as in the case $\delta=\kappa$, saturation of $\mathbf{C}\left[E_{0}\right]$ : In $\left(M^{n+1}\right)^{P_{\kappa} * Q_{A_{n}}}, \mathbf{C}\left[E_{n}\right]$ is saturated; hence in $\left(V_{1}\right)^{Q_{A_{n}}}, \mathbf{C}\left[E_{n}\right]$ is saturated; hence in $V_{1}^{Q_{A_{\omega}}}, \mathbf{C}\left[E_{n}\right]$ is saturated.

When $\kappa$ is a measurable cardinal of a successor order, say $\gamma+1$, then while constructing the set $A_{\gamma}$ we construct on the side $V_{1}^{Q}$-names for conditions in $j^{\gamma+1}\left(Q_{A \cap \alpha}\right)$, to guarantee saturation of the filter $\mathbf{C}\left[E_{\gamma}\right]$.

\section{REFERENCES}

1. J. Baumgartner, A. Taylor and S. Wagon, On splitting stationary subsets of large cardinals, J. Symbolic Logic 42 (1976), 203-214.

2. T. Jech, Stationary subsets of inaccessible cardinals (J. Baumgartner, ed.), Proc. Summer Research Conf. in Math. Sci., Boulder, Col., 1983, Contemporary Math., vol. 31, Amer. Math. Soc., Providence, R. I., 1984, pp. 115-142.

3. K. Kunen, Some applications of iterated ultrapowers in set theory, Ann. Math. Logic 1 (1970), 179-227.

4. __ Saturated ideals, J. Symbolic Logic 43 (1978), 65-76.

5. K. Kunen and J. Paris, Boolean extensions and measurable cardinals, Ann. Math. Logic 2 (1971), 359-378.

6. W. Mitchell, Sets constructible from sequences of ultrafilters, J. Symbolic Logic 39 (1974), 57-66.

7. _ The core model for sequences of measures, Math. Proc. Cambridge Philos. Soc. (to appear).

8. S. Shelah, Proper forcing, Lecture Notes in Math., vol. 940, Springer Verlag, Berlin, 1982.

9. R. Solovay, Real-valued measurable cardinals, Axiomatic Set Theory (D. Scott, ed.), Proc. Sympos. Pure Math., vol. 13, Part 1, Amer. Math. Soc., Providence, R. I., 1971, pp. 397-428.

Department of Mathematics, The Pennsylvania State University, UniverSITY PARK, PENNSYLVANIA 16802

Department of Mathematics, California institute of Technology, PasaDENA, CALIFORNIA 91125 